\title{
Development of Surgical and Visualization Procedures to Analyze Vasculatures by Mouse Tail Edema Model
}

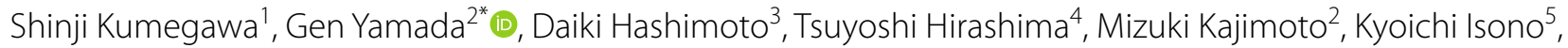
Kota Fujimoto ${ }^{2}$, Kentaro Suzuki ${ }^{2}$, Kazuhisa Uemura' ${ }^{1}$, Masatsugu Ema ${ }^{6}$ and Shinichi Asamura ${ }^{1}$

\begin{abstract}
Background: Because of the high frequency of chronic edema formation in the current "aged" society, analyses and detailed observation of post-surgical edema are getting more required. Post-surgical examination of the dynamic vasculature including L.V. (Lymphatic Vasculature) to monitor edema formation has not been efficiently performed. Hence, procedures for investigating such vasculature are essential. By inserting transparent sheet into the cutaneous layer of mouse tails as a novel surgery model (the Tail Edema by Silicone sheet mediated Transparency protocol; TEST), the novel procedures are introduced and analyzed by series of histological analyses including video-based L.V. observation and 3D histological reconstruction of vasculatures in mouse tails.
\end{abstract}

Results: The dynamic generation of post-surgical main and fine (neo) L.V. connective structure during the edematous recovery process was visualized by series of studies with a novel surgery model. Snapshot images taken from live binocular image recording for TEST samples suggested the presence of main and elongating fine (neo) L.V. structure. After the ligation of L.V., the enlargement of main L.V. was confirmed. In the case of light sheet fluorescence microscopy (LSFM) observation, such L.V. connections were also suggested by using transparent 3D samples. Finally, the generation of neo blood vessels particularly in the region adjacent to the silicone sheet and the operated boundary region was suggested in 3D reconstruction images. However, direct detection of elongating fine (neo) L.V. was not suitable for analysis by such LSFM and 3D reconstruction procedures. Thus, such methods utilizing fixed tissues are appropriate for general observation for the operated region including of L.V.

Conclusions: The current surgical procedures and analysis on the post-surgical status are the first case to observe vasculatures in vivo with a transparent sheet. Systematic analyses including the FITC-dextran mediated snap shot images observation suggest the elongation of fine (neo) lymphatic vasculature. Post-surgical analyses including LSFM and $3 \mathrm{D}$ histological structural reconstruction, are suitable to reveal the fixed structures of blood and lymphatic vessels formation.

Keywords: Lymphedema, Lymphatic vasculature, Tail model, Live imaging, Transparent sheet, 3D reconstruction, Light sheet fluorescence microscopy(LSFM)

*Correspondence: genyama77@yahoo.co.jp; gensan7@wakayama-med.ac.jp ${ }^{2}$ Department of Developmental Genetics, Institute of Advanced Medicine, Medical University of Wakayama, Wakayama, Japan

Full list of author information is available at the end of the article

\section{Background}

Onset of chronic edema in cancer and geriatrics is getting more reported these days.

Presence of the complex vascular network structures has delayed observation of L.V. and blood vessels. Such observation is particularly required to analyze the original author(s) and the source, provide a link to the Creative Commons licence, and indicate if changes were made. The images or other third party material in this article are included in the article's Creative Commons licence, unless indicated otherwise in a credit line to the material. If material is not included in the article's Creative Commons licence and your intended use is not permitted by statutory regulation or exceeds the permitted use, you will need to obtain permission directly from the copyright holder. To view a copy of this licence, visit http://creativecommons.org/licenses/by/4.0/. The Creative Commons Public Domain Dedication waiver (http://creativeco mmons.org/publicdomain/zero/1.0/) applies to the data made available in this article, unless otherwise stated in a credit line to the data. 
aberrant vascular structures in pathogenic conditions [13]. Among such pathogenesis, edema formation is noted as abnormal changes of L.V. (Lymphatic Vasculature). Accumulation of tissue fluids, which frequently includes various inflammatory products, is a prominent factor leading to form edema. Such accumulation can be caused by aberrant reduction of liquid drainage or augmented production of tissue fluids. It has been known that edema formation hampers the QOL of patients frequently leading to accelerated inflammatory reactions. Post-surgical edema formation in various malignant cancers including prostates and breasts is increasing in the modern "aged" society. Surgical removal of target tissues including prostatic and breast region often leads to the adjacent tissue damage and removals of lymphatic structures depending on their types on malignancy. After such procedures, edema is gradually formed due to the aberrant disorganization of L.V. in patients [4-6]. Thus, precise observation and analysis of the vascular structures are particularly essential to treat chronic edema.

In order to treat and cure edema in patients, analysis of the causative pathological incidents is essential by using experimental animal models. Generally, animal models have been developed to improve many analyses on vascular, developmental studies [7-9]. To proceed analysis for model animal studies, detailed observation of the dynamic vasculature in the target tissue areas is essential. Hence, analytical procedures of L.V. and blood vessels in post-operative surgeries in mouse models are becoming more important in vessel biology and surgical methodology fields.

Abnormal connections including neo-vasculature formation in L.V. have been suggested in edematous conditions. Observation of L.V. and blood vessels requires sophisticated visualization procedures. Previous works have been increasing in the medical biology of L.V. for pathogenic conditions and cancer [10-12]. To get breakthrough for the analysis of edematous condition, efficient live conditioned analytical procedure of the operated area is essential.

It has been generally speculated that fine (neo) L.V. has been generated particularly for the operated area which are expected to play roles for the gradual decrease of edematous conditions as recovery processes [13]. Visualization procedures through various dye staining has hitherto been applied to observe vasculatures associated with edema including L.V. and blood vessels. In order to get advantages in such visualization processes, a novel surgical procedure was developed as the Tail Edema by Silicone sheet mediated Transparency protocol (TEST) allowing L.V. observation. The method utilizes the transparent silicone sheet insertion above the blood vessels and the L.V. in the mouse tail to observe of the live vascular tissues in vivo. By utilizing the video through a binocular microscope, it is feasible to observe L.V. after its surgery. In order to evaluate the surgical procedures and several visualization processes, we also examined different types of visualization procedures post-surgically in the mouse tail edema model. In order to perform other visualization analysis of the L.V, light sheet fluorescence microscopy(LSFM)and 3D histological image reconstruction after processing the fixed tail tissue by transparent kit (CUBIC system) were utilized. We performed series of histological analysis using 3D reconstruction of vasculature structures in mouse tails. Introduction of the new TEST procedures with visualization techniques are discussed as novel strategies to analyze post edematous conditions which are expected to improve strategies to treat post-surgical edemas.

\section{Results}

\section{Development of the Post-Surgical Tail Edema by the TEST Procedure}

Practical procedures for the TEST model were developed in the current study. As shown in Fig. 1 and 2A, $0.5 \mathrm{~mm}$ thin transparent silicone sheet (Tigers Polymer, Osaka, Japan) was carefully inserted and sutured with 7-0 nylon to the operated site ( $5 \mathrm{~mm}$ square) in the mouse tail (See Methods). By the current experimental protocols, detailed post-surgical analysis of the vasculatures in the operated area is possible.

To examine the time course and conditions of postsurgical edema formation, sequential observation of the swelling status of treated tail was shown (Fig. 1B). In this procedure, it specifically ablates L.V. restoring the cutaneous layer of the operation site preventing side effects in post-surgical conditions by the sheet insertion.

Immediately after the surgical treatment of TEST, no prominent swelling was observed between POD0 (postoperated day 0) and POD3 (data not shown). As seen in the photos of treated tails, no abnormal bleeding nor inflammatory reactions were observed. To show such plateau status of the swelling, fixed tail location showing the gradual increase (POD0-21) and plateau (POD21-35) status were marked with dots (Fig. 1B). The calculation of the edematous tail volume was performed following the previous reports [14]. As observable by the graph of tail volumes, prominent swelling was maintained between POD21-35.

Additional wounding reaction and necrosis were rarely observed post-surgically. Compared with the previous simple surgical procedure to obtain edema formation (such as 10 days - 2 weeks of time) $[15,16]$, the current procedure enabled longer time of edema observation. Altogether, the current TEST procedure yielded reliable chronic edema formation post-surgically at higher 


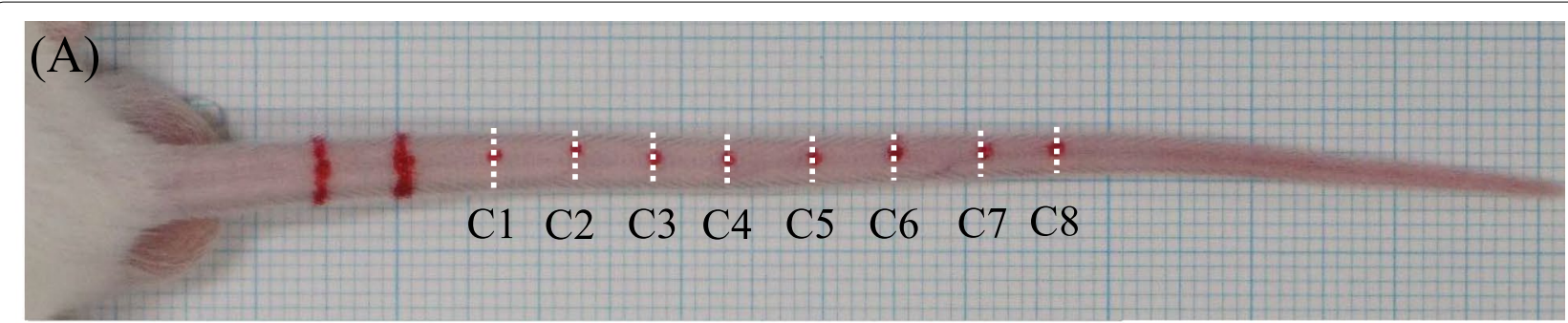

\section{(B)}

\section{tail volume}

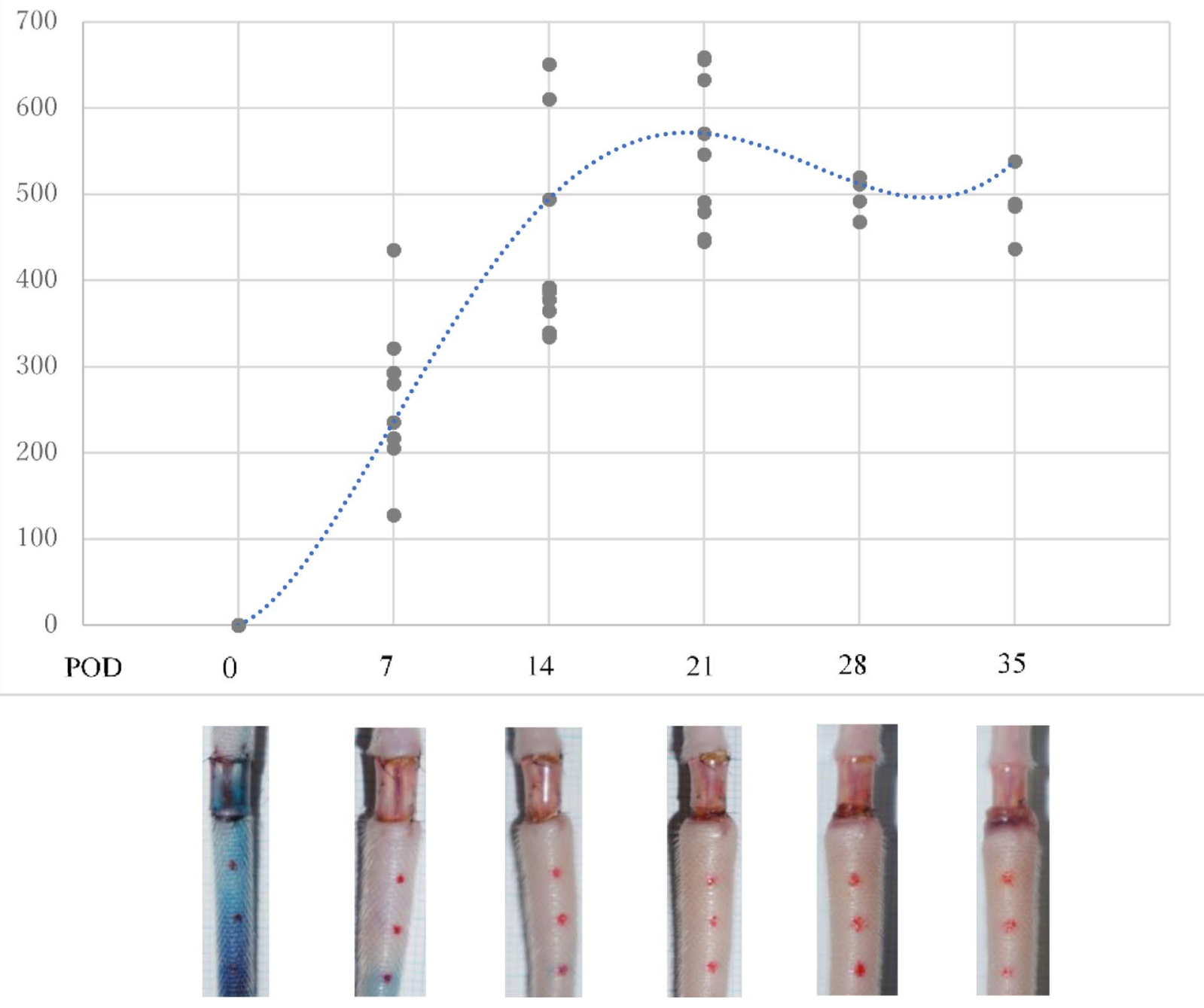

Fig. 1 Parameters for post-operative tail volumes were shown by the calculations as previously reported (by using the parameters (C1, C2,...., C8) in Fig. 1A) [14]. Figure 1B shows the sequential observation of the swelling status of TEST-operated mouse tail with the illustration for the volume calculations. PODO (post-operated day 0) and the gradual increase (POD0-21) and plateau (POD21-35) points of the operated tails were shown (Fig. 1B) 
frequency. Hence, the TEST can be utilized for chronic lymphatic edema formation rather than observing the acute phase of edema formation. Due to the obstruction of lymphatic structures at the operation site, analysis on the subsequent changes of adjacent lymphatic structure is the main concern (see below).

\section{Visual Inspection for the Operated Vasculatures}

In the case of human clinics, either PB (Patent blue dye) or ICG (Indocyanine green dye) were utilized for the inspection for the operated site $[17-20]$. PB mediated visualization has been often utilized for evaluating various surgical procedures as it is stable for several manipulations and visible in optical wavelength light. Although application of $\mathrm{PB}$ is a feasible procedure, detection of fine (neo) vasculature system is often not possible due to its relatively low sensitivity by optical wave length-light. Hence, visualization and in vivo imaging by various dye injection is becoming frequently reported [21-24]. Injected PB after the current procedure showed the successful ligation and enlargement of the main L.V. (Additional file 3B, see Methods). To get advantages of the current transparent sheet application with fluorescent dye mediated visualization, FITC (Fluorescein isothiocyanate)-dextran (FD2000s, 60,842-46-8, SIGMA) was injected $2 \mathrm{~cm}$ peripherally from the operation site in subcutaneous dorsal aspect of tail (Fig. 2A). After confirming the plateau level of edema formation (Fig. 1, POD21-35), vascular analysis was performed to examine the elongation of neo-vascular formation related with the gradual recovery of edema. To observe such condition, the FITC-dextran was injected peripherally from the operation site and dynamic vasculature formation in the operated area through transparent window was examined in live conditions at POD21. Figure 2, (1)-(6) show the rapid distribution of the injected FITCdextran; absence of the FITC-dextran signal before the injection (1), the subsequent rapid distribution of FITCdextran along with the main L.V. after $0.1 \mathrm{~s}$ of injection (2), significant accumulation of FITC-dextran induced by the ligation site after $0.3 \mathrm{~s}$ of injection (white arrow, 3), appearance of the fine (neo) L.V. along with the main L.V. (shown by white dotted arrow after $0.5 \mathrm{~s}, 4$ ), decreased FITC-dextran signal in the upper fine (neo) L.V. and reduced level of the FITC-dextran accumulation at main
L.V. (5) and disappearance of the FITC-dextran signal in L.V. after $1 \mathrm{~s}$ (6). An experimental illustration shows the distribution of the FITC-dextran in the main L.V. and fine (neo) L.V. connections (Fig. 2A). The injection needle is placed adjacent to the L.V.s so that such subcutaneously injected FITC-dextran reaches to such L.V.s. Such observations suggest the development of fine (neo) L.V. formation adjacent to the L.V. structure (Lower part of Fig. 2A).

In order to further demonstrate the efficacy of visualizing the elongating fine structure, plotting of the elongated L.V. branches was shown. Detection of elongating fine (neo) L.V. by snapshot images was shown after TEST procedure. At the time of operation (POD0), elongation was not detected (Fig. 2B). Elongating fine (neo) L.V. was shown at POD7 (Fig. 2C, shown by red arrows, Right side figures are black and white images of the left figures). Further elongation of L.V. was shown by snapshot images after TEST procedure (Fig. 2D). Gradual increase of the post-surgical branched-fine L.V. elongation was shown (Fig. 2B-E) during POD7-14. Significant degree of its increase was suggested (The increase ratio of the branchelongation is shown in the inset graph).

Rapid and dynamic distribution of the injected FITCdextran suggested the post-surgically elomgating L.V. structure. Thus, the current results suggest the potential utility of rapid examination of the fine (neo) and the main L.V. structure during the recovery process of edema formation. After such vasculature elongation was detected, 3D analysis utilizing LSFM and 3D histological reconstruction was performed (see below).

\section{Visualization of the Transparent Post-Operative Tissues by Light Sheet Florescence Microscopy (LSFM)}

Procedures to generate tissue transparency are getting advanced these days [25-30]. To achieve precise observation of vasculatures by the fixed transparent operated regions, Clear, Unobstructed Brain/Body Imaging Cocktails (CUBIC) Protocol II mediated observation was performed [31, 32]. LSFM is generally useful to observe rather large transparent samples (Fig. 3 (A)) [27, 33]. After tissue transparency processing, FITC-dextran injection and subsequent microscopy observation is possible with LSFM.

In this study, we used a $2 \mathrm{mg} / \mathrm{ml}$ solution of tetramethylrhodamine-conjugated lysine fixable dextran of

(See figure on next page.)

Fig. 2 Fig. 2 shows images of a computer screen observed with a fluorescence microscope, taken shots at 60i 17 M (FH), $1920 \times 1080$ (60i), average bit rate $17 \mathrm{Mbps}$ and 30 frames per second, and snapshots were extracted by the movie. Dynamic L.V. formation in the operated area through transparent window was suggested in live conditions at POD21. (1)-(6) by the rapid distribution of the injected FITC-dextran (green). An experimental illustration shows the distribution of the FITC-dextran in the main L.V. and fine (neo) L.V. and the connections (A). In order to further demonstrate the efficacy of visualizing the elongating fine L.V. structure, plotting of the elongated branches was shown. Gradual increase of the post-surgical branched-fine L.V. elongation was plotted (B, C, D and E) during POD7-14. Rather stable degree of the its increase ratio was suggested (inset graph) 

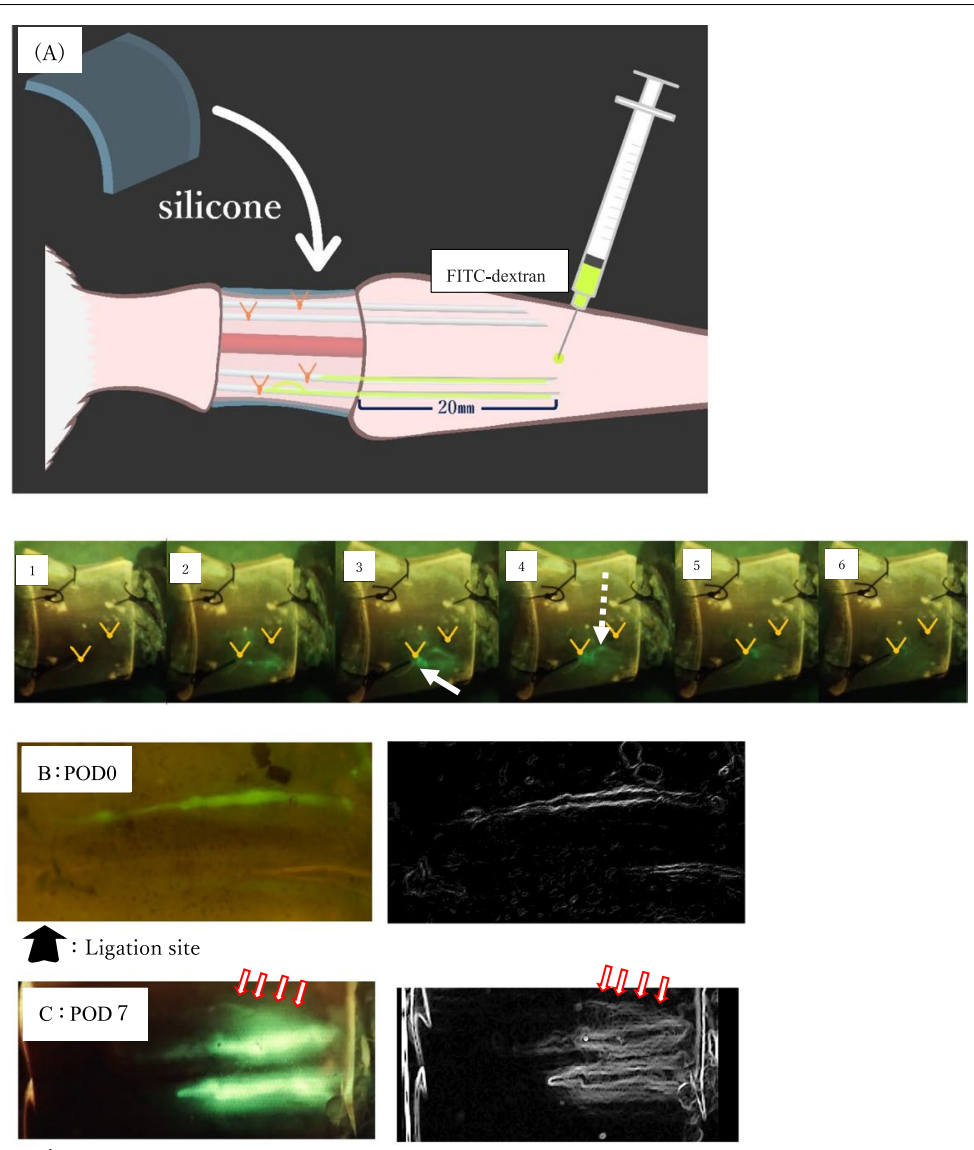

U: Ligation site

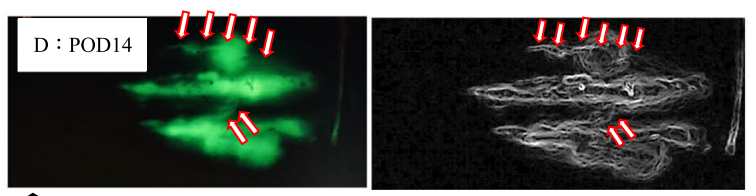

W : Ligation site

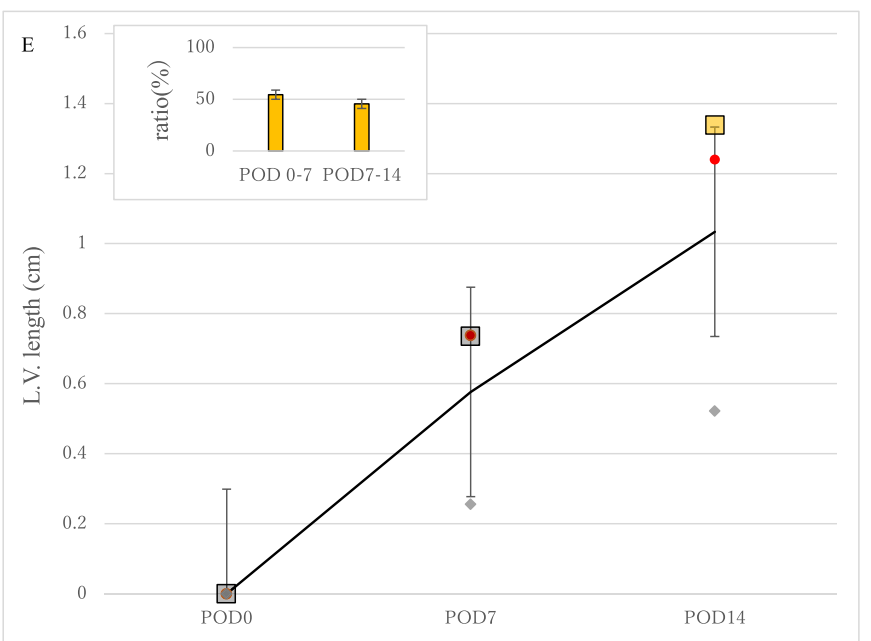

Fig. 2 (See legend on previous page.) 
(A)

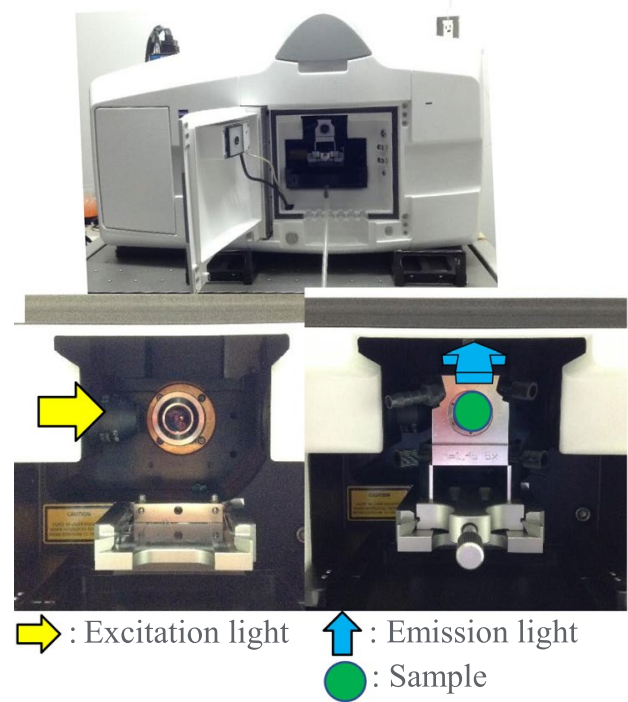

(B)

Boundary of the edema region

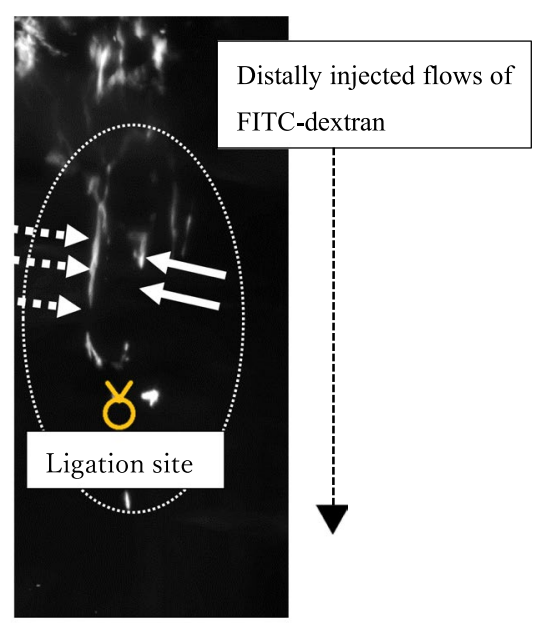

Toward the base of the tail

Fig. 3 Fluoresce images were acquired with light sheet fluorescence microscopy (LSFM), (ZEISS Z.1, Germany). The LSFM system set up is shown (A). PFA fixed specimen was placed at sample fold (green circle) with Excitation light (yellow arrow) toward the detection device by Emission light (blue arrow). The distribution of FITC dextran signal was suggested the presence of main L.V. (shown by two white arrow) and presence of presumptive fine (neo) L.V. (shown by white dotted arrow in (B)). It was suggested that main and fine (neo) L.V. tended to extend to the ligation site (yellow mark, (B))

$2000 \mathrm{kDa}$ (Invitrogen, Carlsbad, CA) to fix its distribution in the L.V. and observed it by LSFM [34]. Figure 3 (B) shows structures of L.V. shown by the transparent region. By such LSFM data, white line (candidate signal for main L.V. shown by white arrows) appeared to extend to the ligation site (yellow mark) (Fig. 3 (B)). In addition, elongated and curved fine (neo) L.V. was also suggested (candidate fine (neo) L.V. shown by white dotted arrows). Other LSFM picture also showed the presence of branched bud-like L.V. structure at POD14 (Additional file. 1). Taken together, the presence of new L.V. in the ligated areas of L.V. was suggested.

\section{D Histological Image Reconstruction Showing the Lymphatic Vasculatures and Blood Vessels by the TEST Protocol}

Transparent sample analysis for visual observation is getting more reported in medical science [25, 27, 35-41].

$3 \mathrm{D}$ histological reconstruction technique is valuable to reveal the tissue structure [42-45]. In order to characterize the pathogenesis induced by the current surgical model, 3D reconstruction of post-surgical histological images was constructed. Total number of 533 serial section of fluorescent immunostaining images were incorporated as digital data and processed by Amira software to construct 3D images of edema and its adjacent operated regions (Fig. 4 (A)-(D)).

Cross-section of the TEST operated specimens (Fig. 4 (C), (D)).

The area corresponding to the $3 \mathrm{D}$ image is indicated by the red square line (Fig. 4 (A)). The main L.V.s (white colored) run along the sides of the tail and the lateral veins (blue colored) and its distribution is also shown (Fig. 4 (B)). The ventral artery runs along the tail (Fig. 4 (B): red colored).

By series of 3D movies (see Additional file 2), the next observations were obtained.

Toward the base of the tail in the operated region, generation of fine blood vessel was observed by green signal (Fig. 4 (C): dotted white circle region). Altogether, the prominently developed blood vessels at the peripheral edge of the silicone sheet, and underneath of it (Fig. 4 (C): dotted white circle region) were observed. In contrast, generation of new L.V. was not confirmed in such region (absence of prominent purple signals in dotted white circle). Due to the ligation of main L.V., it may be possible that new L.V. generation was not prominent.

In the operated region adjacent to the edema, the presence of two main enlarged L.V. by surgical ligation was clearly observed (Fig. 4 (D); the ligation site was shown by yellow circle). Associated with the TEST, fine hyperplasic 


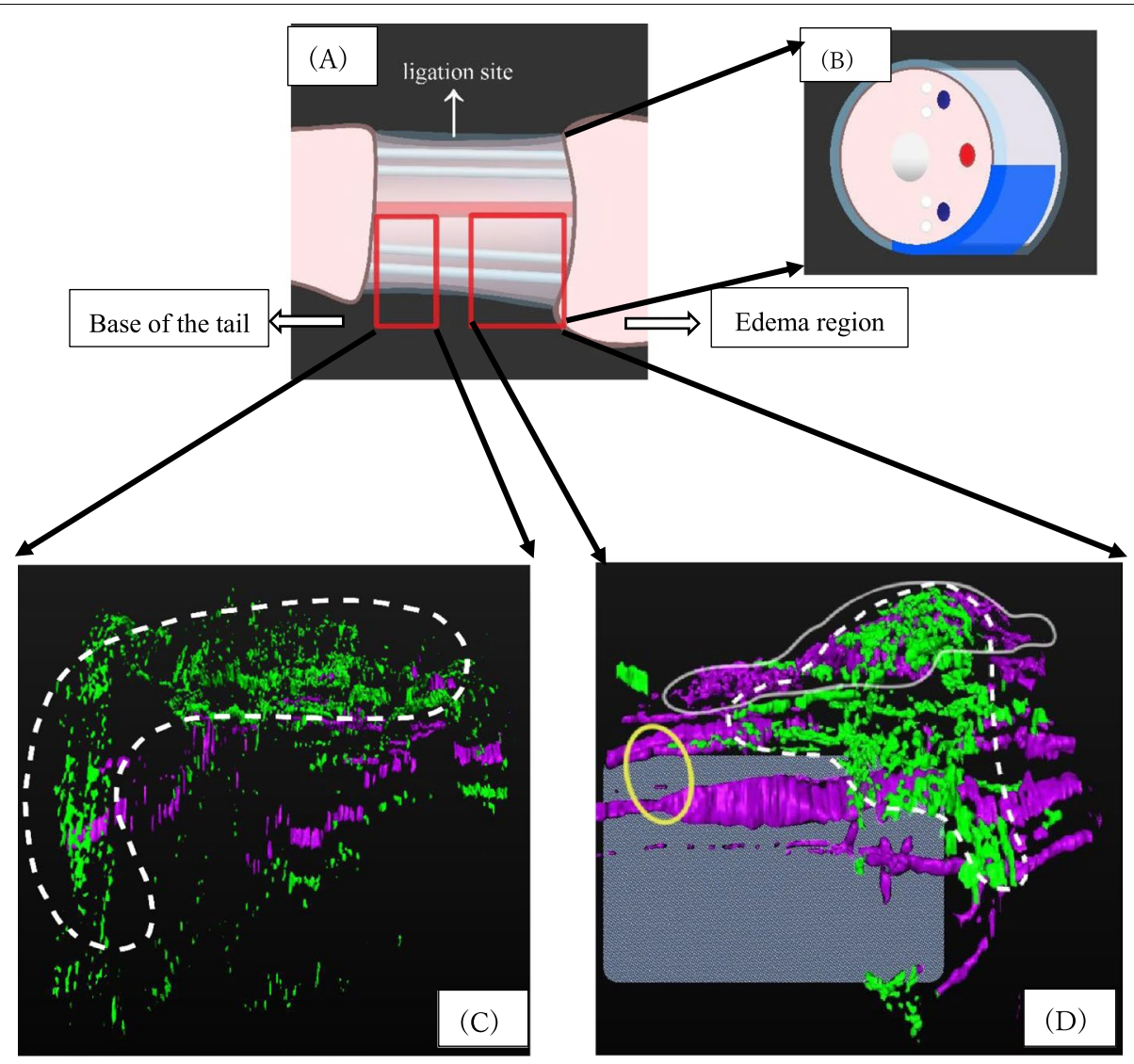

Fig. 4 The area corresponding to the 3D image is indicated by the red square line (A). The main L.V.(white colored) runs along the sides of the tail and the lateral veins (blue colored) and its distribution is also shown (B). The ventral artery runs along the tail ((B): red colored). Total number of 533 serial sections of fluorescent stained (either CD31 or LYVE1) images were incorporated as digital data and processed by Amira software to construct 3D images of edema and its adjacent regions (C, D). In more proximal side from the operated region (adjacent to the base of the tail), generation of fine blood vessel was observed by green signal ((C): in dotted white circle region). As for the operated region close to the edema, the presence of two main enlarged L.V. by surgical ligation was clearly observed ((D); yellow circle region). Fine (neo) hyperplastic L.V. were observed by the purple signals ((D): white circle region), fine (neo) blood vessel formation was observed by the green signals ((D): dotted white circle region)

L.V. were observed adjacent to the cutaneous epithelia next to the operated site and just below the silicone sheet (Fig. 4 (D): purple signal in the white circle region). Such L.V. located adjacent to blood vessels (Fig. 4 (D)). Fine blood vessels (Fig. 4 (D): green signal in dotted white circle region) were also prominent at the distal region of the silicone sheet. In deeper layer of such region, lymphogenesis was not prominently confirmed (Fig. 4 (D): absence of the fine purple signals shown in the grey box region). This indicates the prominent differences of post-surgical lymphatic responses between surface and deep layer of the operated region. Such differential responses of L.V. and blood vessels were suggested for the first time.

\section{Discussions}

Animal Models for Edema and Visualization Analysis In human tumor therapy fields, formation of chronic edema hampers the QOL of patients who received removal of primary cancers [46-48]. Given the highly frequent onset of chronic edema in cancer and geriatrics, animal models of edema are getting more required. It has been reported that estimation and prediction for the onset of post-surgical edema is practically not feasible in many cases even after the careful surgical treatments [4, 49]. Examination of such chronic development of postsurgical edema is thus essential which ideally predicts and prevents its onset. Definition of acute and chronic of edema formation has been discussed in experimental animal models and in clinical patients [15, 50-52]. Diagnosis of chronic type of lymphedema is generally determined post-surgically after a few years etc. in patients [50]. Long term edema formation such as weeks of period, is generally referred as chronic types of animal models. Application of transparent sheets by surgical models has been performed to get advantages of its elasticity and stabilize the operated area and prevent its shrinkage [53, 
54]. However, taking advantages of the transparency of the sheet to observe the underlying vessels has not been performed.

By the current TEST procedure, observation of dynamic state of vasculatures is useful to examine the post-operative status. Surgical models of acute and chronic phase of lymphatic edema formation of tail have been modified. In case of mouse hindlimb edema formation, radiation-induced phenotype is often described as chronic type of edema [55-57]. Besides hindlimbs, mouse tail has been often utilized to analyze edema by several surgical procedures. Compared with hindlimb models, tail region is anatomically relatively simple without well-developed muscular structures and its surgical treatment does not necessary lead to impair essential physiological status of mice in laboratory housing conditions. Table 1 summarizes experimental models for tail edema formation listing their experimental advantages and disadvantages. Simple surgical stripping models with ligation of L.V. generally showed outcomes with frequent infection and necrosis (A) [58] . Edema formation model has been originally reported by simple ablation of L.V. such as by electric knife mediated operation (B) [59]. Performing simple invasions for L.V. leads to not only L.V.-mediated fluid accumulation, but also damaging the operated site and underlying dermis [60]. Injuries of cutaneous layers often leads to shrinkable ulcer and abnormal keloid formation in the operated area. Thus, further modification of surgical model is required for specifically modulating vessels by utilizing sheets or collagens with ligating L.V. in the operated area $(C)[16,34,53]$.

Generally, surface cutaneous epithelia and underlying layers including dermis with vasculatures are essential for cutaneous homeostatic regulation. Cutaneous surface modification by various grafting technics has been reported in tissue generation studies [53, 61, 62]. Relatively long period of observation for vasculogenesis in such graft tissues is expected to proceed tissue regeneration studies. Previous non-transparent sheet insertion

Table 1 Summaries of the experimental models for tail edema formation. Historically, simple surgical stripping with ligation of lymphatic vesicle yielded not only experimental edema but frequent infection and necrosis (A). Ablation by electric knife mediated operation improved the efficiency of operation but often damaged the nearby tissues (B). Formation of edema by simple invasion of lymphatic vesicle frequently damaged operated site and the nearby dermis. Modification of the surgical procedures with various experimental non-transparent sheets or collagen have been performed (C). Transparent sheet insertion of the operated area allows visual inspection reducing the post-surgical side effects (D).

\begin{tabular}{|c|c|c|c|c|c|}
\hline & $\begin{array}{l}\text { Protocols of surgical } \\
\text { procedures for edema }\end{array}$ & $\begin{array}{l}\text { Surgical treatment } \\
\text { for L.V. }\end{array}$ & Duration of edema & $\begin{array}{l}\text { Advantages and } \\
\text { disadvantages as } \\
\text { experimental models }\end{array}$ & References \\
\hline (A) & $\begin{array}{l}\text { Procedures of stripping surface } \\
\text { skins, cutaneous layers }\end{array}$ & The ligation of L.V. & $\begin{array}{l}\text { Peaks of edema } \\
\text { formation (14days) }\end{array}$ & Frequent infection and necrosis. & $\begin{array}{l}\text { Zampell JC, et al } \\
{[58]}\end{array}$ \\
\hline (B) & Full-perimeter incision & $\begin{array}{l}\text { Procedures including } \\
\text { electric knife treatment }\end{array}$ & $\begin{array}{l}\text { Peaks of edema } \\
\text { formation (24days) }\end{array}$ & Simple procedures of surgeries & Tian W.[59] \\
\hline (C) & $\begin{array}{l}\text { Modified stripping procedures } \\
\text { with various sheets, collagens }\end{array}$ & The ligation of L.V. & $\begin{array}{l}\text { Peaks formation of } \\
\text { edema (such as } \\
\text { 10days, 25days) }\end{array}$ & $\begin{array}{l}\text { Modification of collagen or } \\
\text { tegaderm treatment and placing } \\
\text { anchoring site for fine (neo) } \\
\text { L.V. formation, reducing the } \\
\text { operation scar, wound healing } \\
\text { responses. }\end{array}$ & $\begin{array}{l}\text { Clavin NW et al [16] } \\
\text { Uzarski J, et al [34] } \\
\text { Jimi S, et al [53] }\end{array}$ \\
\hline (D) & $\begin{array}{l}\text { Procedures utilizing transparent } \\
\text { silicone insertion (TEST) }\end{array}$ & $\begin{array}{l}\text { Ligation, fine treatment } \\
\text { of the skin with } \\
\text { transparent silicone sheet }\end{array}$ & $\begin{array}{l}\text { Peaks of edema (3- } \\
\text { 4weeks after } \\
\text { surgery) and chronic } \\
\text { edema formation }\end{array}$ & $\begin{array}{l}\text { Procedures for visualization of } \\
\text { lymphatic, L.V. vasculatures } \\
\text { without inducing scar and } \\
\text { wound healing was delayed. }\end{array}$ & The current study \\
\hline
\end{tabular}


surgeries only prevented the rapid onset of wounding and scar formation. In case of non-transparent silicone application, it was also utilized for surgical operation of stoma reconstructing its efficient functions [63, 64]. In that aspect, the current transparent sheet application is regarded as one of the first silicone-mediated procedures for visualization and improving stability of the operated area.

\section{Dynamic Status of Fine (Neo) - Main L.V. Formation in the Operated Site of TEST}

Accurate ligation of L.V. and subsequent replacement by transparent silicone sheet (TEST) is reported in the current study. Insertion of transparent silicone allows observation of chronic edema formation. By this insertion, observation underneath of the silicone sheet in vivo becomes possible using fluorescent dye injection. Surgical ligation was confirmed by the $\mathrm{PB}$ injection staining (Additional file 3A, B). Rapid fluorescent staining and the snapshot observation from the main and fine L.V. suggested the presence of dynamic post-surgical L.V. formation (white dotted arrow in Fig. 2: (1)-(6)). Furthermore, examination for the extent of such fine L.V. elongation indicated the effective visualization of the transparent sheet mediated lymphogenesis. It is still speculative whether such newly developed connections were initiated adjacent from the main L.V. ligation site or not. Due to such ligation, increased volume and pressure were assumed as generated not only in the ligation site but along with the main L.V. It is thus possible that interconnection of such main - fine (neo) L.V. was dynamically generated close to ligation site. In case of human cancer clinics, after resection of primally tumor such as at breast/ prostate, careful ligation of several lymphatic nodes and vasculature was extensively performed [65-67]. Attention has been focused on the completeness of ligation or electric knife treatments as an efficient surgical procedure, but less attention has been focused on its subsequent effects in the adjacent regions. Hence, detailed analysis for the effect of ligation adjacent to the operated site is essential and the current analysis is one of the initial works showing dynamic changes of vasculature.

Treatments including post-surgical reconstructive surgery, self-drainages etc. may generally augment the generation of main - fine (neo) L.V. connections possibly reducing the extent of post-surgical edema. Hence, the current TEST procedure can be combined for various post-surgical analysis including various treatments. Connections between pre-existent L.V. and fine (neo) L.V. structures need to be analyzed also with L.V. generating activity [68]. IL6 (Interleukin6) has been one of the major factors regulating inflammation and also lymphogenesis [69]. Various factors affecting lymphogenesis such as IL6 can be applied in this TEST procedure related experiments $[69,70]$. The finding of post-surgical connections of main - fine (neo) L.V. may open a research field for the post-surgical edema. Further analysis for the effect of surgical ligation can be studied by utilizing live vasculature images plus observation by detailed 3D analysis.

As for other post-operative visualization, comparisons for transparent analysis for LSFM observation was shown in the current study. LSFM is capable of imaging samples spanning orders of magnitude in space and time and it is suitable for observing transparent samples because of its optical depth focus and scanning characters [33]. Because general optical light depth by LSFM is around $6-8 \mathrm{~mm}$, it can cover various fine (neo) L.V. around the ligation site in this experimental set up. Both LSFM and 3D imaging method possesses advantages and disadvantages. In the case of 3D histological reconstruction, long process of histological section preparation, staining and subsequent reconstruction are necessary. After performing such process, information of L.V. and blood vessels can be analyzed with CD31 (endothelial marker) and LYVE1 (L.V. endothelial marker) staining data. In the case of LSFM observation, tissue processing including transparent tissue preparation is still necessary. However, taking images after injection of FITC-dextran is sensitive without procedures of sectioning. Utilizing lysine conjugated FITC-dextran is essential, because of its retention inside the L.V. after fixation. Such fixable fluorescent dye enables to show the relatively longer fluorescent dye distribution between main- fine (neo) L.V. For both LSFM and $3 \mathrm{D}$ reconstruction analysis, continuous observation of the elongation proses of L.V. is not possible because of the fixation for observing sample. Because of the significant experimental time required for experiments and inability of continuous observation after fixation, the current type of visual binocular snapshot analysis through transparent sheet by TEST procedure will be particularly useful as the first experimental protocol.

\section{Conclusion}

The new surgical procedures and analysis on the postsurgical edematous status of mouse models are introduced in the current study. Formation of tail edema by transparent sheet insertion was analyzed (TEST surgical procedure). Live images taken after the procedure suggested the presence of main and elongating fine (neo) L.V. formation for the first time. In addition to such live imaging, two histological observation experiments were performed.

Light sheet fluorescence microscopy (LSFM) mediated observation indicated the presence of fine L.V. formation. 
3D histological image reconstruction also showed the fine structure of blood and lymphatic vessels adjacent to the edema region and in the surface cutaneous region. However, such two procedures can be performed only by fixation of post-surgical samples thus, not suitable for imaging for the L.V. elongation in vivo.

\section{Methods}

\section{A Novel Lymphedema Mouse Tail Model (TEST)}

We developed the mouse tail surgery of lymphedema as a new procedure (TEST) in this study. Anesthesia was performed by administering three types of mixed general anesthesia (Medetomidine $(0.3 \mathrm{mg} / \mathrm{kg}$ ), Midazolam $(4 \mathrm{mg} / \mathrm{kg})$, Butorphanol $(5 \mathrm{mg} / \mathrm{kg}))$ into abdominal cavity of mice. In the model, a $5 \mathrm{~mm}$ wide circumferential full-thickness skin section was excised $2 \mathrm{~cm}$ distal to the tail base to remove superficial L.V. The procedures were designed so that the vessels were clearly visible underneath the transparent silicone sheet (Additional file 3A). Improper visualization was judged by compressing blood vessels with fine forceps to avoid the central region of the surgical field was stagnant or the operated blood vessel was visible only in part of the transparent window region. A yellow arrow shows smooth distribution of endogenous blood along with the L.V. after the TEST surgery (Additional file 3A).

After patent blue (PB) was administered subcutaneously from the distal part of the tail to identify the position of deep L.V.s, they were extensively ligated with 10-0 nylon suture. After performing incision of the operated area of skin, examination of the location of L.V. and vein underneath skin and subsequent peeling off the L.V. was performed without inducing bleeding. Next, an examination was performed so that the injected PB did not show proximal leakage adjacent to the ligation and the L.V.s were expanded at the operation site (Additional file 3B: white dotted circles). A $5 \mathrm{~mm}$ wide rectangular silicon splint was fabricated from a $0.5 \mathrm{~mm}$ thin silicon sheet (125-18-11-01, Tigers Polymer, Osaka, Japan). Such silicone materials were selected by its transparency and flexibility as an insert membrane. The transparent silicone sheet was originally utilized for coverage of PC keyboard to secure transparent operation with flexibility and strength.

The transparent silicone sheet should clarify the next conditions of TEST surgical operation.

1. Flexible and solid as surgical material

2. Stable and durability as material for surgical operations including suture

3. Fitness and adhesiveness to the operation area, the curved structure of mouse tail
The silicone splint was sutured to the skin at both edges of the skin-peeled surgical field with 7-0 nylon, and the surgical procedure was completed. The silicone was ligated from the 0 o'clock direction (dorsal side of the tail) to avoid the blood vessels. To avoid excessive pressure on the blood vessels, the length of the silicone was finally adjusted ligating to the tail.

\section{Calculation of the Edema Status}

The kinetics of edema formation after post-surgical TEST procedure were shown (Fig. 1). The degree of edema formation was calculated weekly using the truncated cone formula: $\mathrm{V}=\mathrm{l} / 4 \pi(\mathrm{C} 1 \mathrm{C} 2+\mathrm{C} 2 \mathrm{C} 3 \ldots+\mathrm{C} 7 \mathrm{C} 8)$ [14]. Tail diameters were measured using digital calipers every $0.5 \mathrm{~cm}$ starting from the surgical site going distally toward the tip of the tail (Fig. 1A). The time point of postoperative day was indicated as POD including POD0 as the time of the operation. Each point in the graph represents increased tail volume compared with POD0 for each operated specimen (Fig. 1B). During POD0-21, gradual increase of edema formation was revealed by the tail volume and inspection of the corresponding swelling status (lower part of Fig. 1B). During POD 21-35, the plateau state of such edema formation was observed.

\section{Animals}

Male ICR mice (2 months old) were purchased from CLEA Japan. All procedures and protocols have been approved by Animal Research Committee of Wakayama Medical University (approval number: 994). We have confirmed that all methods were carried out in accordance with relevant guidelines and regulations.

\section{Dynamic Video Recording of Binocular Microscope Images after FITC-Dextran Injection}

In order to take sequential snapshot images of FITCdextran (2000 kDa, 60,842-46-8, SIGMA) injected L.V. images, FITC-dextran was injected subcutaneously into $2 \mathrm{~cm}$ peripherally from the operation site by administering three types of mixed general anesthesia (Medetomi-dine, Midazolam, Butorphanol) into abdominal cavity (ICR mice; $N=9$ ). Immediately after the injection (approximately till $5 \mathrm{~s}$ ), video images were taken by binocular fluorescent microscope (Leica: M165 FC). Snapshot FITC images were shown at the interval of $0.5 \mathrm{~s}$ immediately after injection. Original video images were recorded by the form of MTS files and snapshots were extracted by the movie.

In addition to the tail volume measurement at each post-surgical points, increase of several stages of tail L.V. length was calculated between POD0-7 and POD7-14 (Fig. 2B-E). The increase ratio (shown as POD0-7 value by POD0-7/ POD0-14) was shown by the inset graph. 


\section{CUBIC Protocol for Processing the Fixed Tail Tissue for Transparency}

The entire tails were fixed overnight in $4 \%$ paraformaldehyde (PFA) in Phosphate- buffered saline (PBS). The fixed tails were harvested $5 \mathrm{~mm}$ centrally and $10 \mathrm{~mm}$ peripherally from the surgical site and then washed with PBS overnight at room temperature (about $25^{\circ} \mathrm{C}$ ). The samples were immersed in a 1:1 mixture of CUBIC-L solution (T3740, Tokyo Kasei Kogyo) and PBS for 1 day at $37^{\circ} \mathrm{C}$ with gentle shaking and CUBIC-L solution at $37^{\circ} \mathrm{C}$ for 2 days with shaking. They were washed with PBS overnight at room temperature. The samples were subsequently immersed in a 1:1 mixture of CUBIC-R solution (T3741, Tokyo Chemical Industry) and PBS with gentle shaking at room temperature for 1 day. The samples were then immersed in CUBIC-R with gentle shaking at room temperature for 2 days.

\section{Light Sheet Fluorescence Microscopy (LSFM) Observation of the L.V. in the Operated Area}

Fluorescence images were acquired with LSFM (ZEISS Z.1, Germany) and the excitation laser $(561 \mathrm{~nm})$ corresponding to FITC-dextran was selected. Samples were immersed in a 1:1 mixture of silicone oil TSF4300 (Momentive Performance Materials, $\mathrm{RI}=1.498$ ) and mineral oil (Sigma-Aldrich, $\mathrm{RI}=1.467$ ) during image acquisition [31]. We prepared a customized sample holder for capturing the image of operated area. A fluorinated ethylene-propylene (FEP) tube with a refractive index almost equal to that of water was used to support the sample. The method is to insert rubber into the FEP tube to increase the contact area with the sample. The sample was held in place by applying alonalpha (TOAGOSEI $\mathrm{CO}$, Japan) to the rubber surface. In order to perform the LSFM imaging, the PFA-fixed specimen was placed at the tissue folder (Fig. 3 (A) shown by green). Excitation light was illuminated (Fig. 3 (A) shown by yellow arrow) and emission light was captured by the sCMOS sensor (Fig. 3 (A) shown by blue arrow, Tokyo Instruments, Inc).

\section{Tissue Processing, Immunostaining and 3D Reconstruction Images}

Tissue samples were fixed in 4\% (wt/vol) PFA overnight, dehydrated in methanol (25-100\%) washes and embedded in paraffin. The paraffin blocks were sectioned serially $(10 \mu \mathrm{m}$ thick) with a microtome (902,100, HM325, PHC, Japan), placed on slides, deparaffinized and rehydrated. Antigen retrieval was performed with either by citrate buffer or by autoclaving at $121^{\circ} \mathrm{C}, 1 \mathrm{~min}$. For primary antibody staining, the following antibodies were utilized: anti-CD31 (endothelial markers; 1/200, AF3628, goat, R\&D systems) and anti-LYVE1 (L.V. endothelial marker; 1/100, ab14917, rabbit, Abcam). For secondary antibody reaction, the following antibody was utilized: Invitrogen Donkey anti-goat IgG Alexa Fluor 488 (1/200, Thermo Fisher Scientific). Total number of 533 serial section of fluorescent immunostaining images were incorporated as digital data and processed by Amira software to construct 3D images of edema and its adjacent regions (Fig. 4).

\begin{abstract}
Abbreviations
L.V.: Lymphatic vasculature; TEST: The tail edema by silicone sheet mediated transparency protocol; LSFM: Light sheet fluorescence microscopy; POD: Post-operated day; PB: Patent blue dye; ICG: Indocyanine green dye; FITC: Fluorescein isothiocyanate; CUBIC: Clear, unobstructed brain/body imaging cocktails; FEP: Fluorinated ethylene-propylene.
\end{abstract}

\section{Supplementary Information}

The online version contains supplementary material available at https://doi. org/10.1186/s12575-021-00159-3.

Additional file 1. Additional file 1 shows another LSFM captured image of bud-like L.V. structure (shown by red circle). Such bud structure was detected at POD14. The ligation suture was remained as a black signal. The main L.V. locates left to the red circle in the picture.

Additional file 2. Additional file 2 2A, 2B In the lower side of such images, 3D reconstructed movie was attached corresponding to $Y$ axis of reconstructed images. Additional file 2A is a 3D movie of Fig. 4(C) Y-axis. The movie shows the prominently developed blood vessels at the peripheral edge of the silicone sheet, and underneath of it. In contrast, generation of fine (neo) L.V. was not confirmed in such region. Additional file $2 \mathrm{~B}$ is a $3 \mathrm{D}$ movie of Fig. 4(D) turning by $Y$-axis. The movie shows fine (neo) hyperplastic L.V. observed adjacent to the cutaneous epithelia next to the operated site and below the silicone. Fine (neo) blood vessels were prominent at the distal edge of the silicone sheet. In deeper layer of such region, lymphogenesis was not prominently confirmed.

Additional file 3. Additional file 3A, 3B Injected PB (Patent Blue dye) after the current procedure showed the successful ligation and enlargement of the main L.V. FITC (Fluorescein isothiocyanate)-dextran (FD2000s, $60,842-46-8$, SIGMA) was injected subcutaneously $2 \mathrm{~cm}$ peripherally from the operation site (3A). $4 \mathrm{~A}$ shows normal and undisturbed distribution of endogenous blood in the operated area (shown by yellow arrow). $3 \mathrm{~B}$ shows the prominent enlargement of two main L.V.s due to the ligation (two white dotted circle area. Ligation was shown by the black suture).

\section{Acknowledgements}

The authors thank Dr. Fumihiko Matsuda, Dr. Yuuki Shimizu, Dr. Yoshitaka Wada, Dr. Satsuki Tachibana and Dr. Kazuki Ueno for their encouragements. The authors also thank all laboratory colleagues for their assistance. We also express our appreciation to Chie Morishita, Yugi Rim and Maya Ueda for their valuable assistance. LSFM imaging was performed at the Medical Research Support Center, Graduate School of Medicine, Kyoto University.

\section{Authors' Contributions}

SK, GY and SA designed the project. SK, DH, TH, MK, KF and KS performed visualization analysis. SK, KI, KU and ME performed mouse experiments. SK and GY prepared the manuscript. All authors read and approved the final manuscript.

\section{Funding}

This work was supported by Japan Society for the Promotion of Science KAKENHI Grants 21 K09772, 21 K09773 and 20 K18415.

Availability of Data and Materials

Not applicable (All data are included in the MS). 


\section{Declarations}

\section{Ethics Approval and Consent to Participate}

Shinji Kumegawa, Gen Yamada, Daiki Hashimoto, Tsuyoshi Hirashima, Mizuki Kajimoto, Kyoichi Isono, Kazuki Ueno, Kota Fujimoto, Kentaro Suzuki, Kazuhisa Uemura, Masatsugu Ema, and Shinichi Asamura declare that they have no conflict of interest. This work does not contain human subjects. All institutional and national guidelines for the care and use of laboratory animals were followed. All procedures and protocols were approved by Animal Research Committee of Wakayama Medical University (approval number: 994).

\section{Consent for Publication}

Not applicable.

\section{Competing Interests}

Not applicable.

\section{Author details}

'Department of Plastic and Reconstructive Surgery, Graduate School of Medicine, Medical University of Wakayama, Wakayama, Japan. ${ }^{2}$ Department of Developmental Genetics, Institute of Advanced Medicine, Medical University of Wakayama, Wakayama, Japan. ${ }^{3}$ Department of molecular Physiology, National Cerebral and Cardiovascular Center, Suita, Osaka, Japan. ${ }^{4}$ The Hakubi Center/Graduate School of Biostudies, Kyoto University, Kyoto, Japan. ${ }^{5}$ Laboratory Animal Center, Wakayama Medical University, Wakayama, Japan. ${ }^{6}$ Department of Stem Cells and Human Diseases Models, Research Center for Animal Life Science, Medical University of Shiga, Otsu, Shiga, Japan.

\section{Received: 17 August 2021 Accepted: 26 October 2021}

Published online: 11 November 2021

\section{References}

1. Kumar DL, DeFalco T. A perivascular niche for multipotent progenitors in the fetal testis. Nat Commun. 2018;9(1):4519.

2. Neufeld S, Planas-Paz L, Lammert E. Blood and lymphatic vascular tube formation in mouse. Semin Cell Dev Biol. 2014;31:115-23.

3. Azimi MS, Motherwell JM, Hodges NA, Rittenhouse GR, Majbour D, Porvasnik SL, et al. Lymphatic-to-blood vessel transition in adult microvascular networks: a discovery made possible by a top-down approach to biomimetic model development. Microcirculation. 2020;27(2):e12595.

4. Cormier JN, Askew RL, Mungovan KS, Xing Y, Ross MI, Armer JM. Lymphedema beyond breast cancer: a systematic review and meta-analysis of cancer-related secondary lymphedema. Cancer. 2010;116(22):5138-49.

5. Warren AG, Brorson H, Borud LJ, Slavin SA. Lymphedema: a comprehensive review. Ann Plast Surg. 2007;59(4):464-72.

6. Petrek JA, Pressman PI, Smith RA. Lymphedema: current issues in research and management. CA Cancer J Clin. 2000;50(5):292-307 quiz 308-211.

7. Hong YK, Shin JW, Detmar M. Development of the lymphatic vascular system: a mystery unravels. Dev Dyn. 2004;231(3):462-73.

8. Oliver G. Lymphatic vasculature development. Nat Rev Immunol. 2004:4(1):35-45.

9. Nagy JA, Vasile E, Feng D, Sundberg C, Brown LF, Detmar MJ, et al. Vascular permeability factor/vascular endothelial growth factor induces lymphangiogenesis as well as angiogenesis. J Exp Med. 2002;196(11):1497-506.

10. Li H, Meng QH, Noh H, Batth IS, Somaiah N, Torres KE, et al. Detection of circulating tumor cells from cryopreserved human sarcoma peripheral blood mononuclear cells. Cancer Lett. 2017;403:216-23.

11. Brix B, Sery O, Onorato A, Ure C, Roessler A, Goswami N. Biology of lymphedema. Biology (Basel). 2021;10(4).

12. Stacker SA, Williams SP, Karnezis T, Shayan R, Fox SB, Achen MG. Lymphangiogenesis and lymphatic vessel remodelling in cancer. Nat Rev Cancer. 2014;14(3):159-72.

13. Mishima T, Ito $Y$, Nishizawa N, Amano H, Tsujikawa K, Miyaji K, et al. RAMP1 signaling improves lymphedema and promotes lymphangiogenesis in mice. J Surg Res. 2017;219:50-60.
14. Sitzia J. Volume measurement in lymphoedema treatment: examination of formulae. Eur J Cancer Care (Engl). 1995;4(1):11-6.

15. Zampell JC, Yan A, Elhadad S, Avraham T, Weitman E, Mehrara BJ. CD4(+) cells regulate fibrosis and lymphangiogenesis in response to lymphatic fluid stasis. PLoS One. 2012;7(11):e49940.

16. Clavin NW, Avraham T, Fernandez J, Daluvoy SV, Soares MA, Chaudhry A, et al. TGF-beta1 is a negative regulator of lymphatic regeneration during wound repair. Am J Physiol Heart Circ Physiol. 2008;295(5):H2113-27.

17. Kung TA, Champaneria MC, Maki JH, Neligan PC. Current concepts in the surgical management of lymphedema. Plast Reconstr Surg. 2017;139(4):1003e-13e

18. Kaplan-Marans E, Fulla J, Tomer N, Bilal K, Palese M. Indocyanine green (ICG) in urologic surgery. Urology. 2019;132:10-7.

19. Wada T, Kawada K, Takahashi R, Yoshitomi M, Hida K, Hasegawa S, et al. ICG fluorescence imaging for quantitative evaluation of colonic perfusion in laparoscopic colorectal surgery. Surg Endosc. 2017;31(10):4184-93.

20. Kim JH, Byeon HK, Kim DH, Kim SH, Choi EC, Koh YW. ICG-guided sentinel lymph node sampling during robotic Retroauricular neck dissection in cNO Oral Cancer. Otolaryngol Head Neck Surg. 2020;162(3):410-3.

21. Li Z, Yao S, Xu J. Indocyanine-green-assisted near-infrared dental imaging - the feasibility of in vivo imaging and the optimization of imaging conditions. Sci Rep. 2019;9(1):8238.

22. Mihara M, Hara H, Araki J, Kikuchi K, Narushima M, Yamamoto T, et al. Indocyanine green (ICG) lymphography is superior to lymphoscintigraphy for diagnostic imaging of early lymphedema of the upper limbs. PLoS One. 2012;7(6):e38182.

23. Ayestaray B, Bekara F, Andreoletti JB. Patent blue-enhanced lymphaticovenular anastomosis. J Plast Reconstr Aesthet Surg. 2013;66(3):382-9.

24. Li Z, Hartzler T, Ramos A, Osborn ML, Li Y, Yao S, et al. Optimal imaging windows of indocyanine green-assisted near-infrared dental imaging with rat model and its comparison to X-ray imaging. J Biophotonics. 2020;13(6):e201960232.

25. Inyushin M, Meshalkina D, Zueva L, Zayas-Santiago A. Tissue transparency in vivo. Molecules. 2019;24(13).

26. Jing D, Yi Y, Luo W, Zhang S, Yuan Q, Wang J, et al. Tissue clearing and its application to bone and dental tissues. J Dent Res. 2019;98(6):621-31.

27. Hirashima T, Adachi T. Procedures for the quantification of whole-tissue immunofluorescence images obtained at single-cell resolution during murine tubular organ development. PLoS One. 2015;10(8):e0135343.

28. Kolesová H, Čapek M, Radochová B, Janáček J, Sedmera D. Comparison of different tissue clearing methods and 3D imaging techniques for visualization of GFP-expressing mouse embryos and embryonic hearts. Histochem Cell Biol. 2016;146(2):141-52.

29. Peeters G, Debbaut C, Laleman W, Monbaliu D, Vander Elst I, Detrez JR, et al. A multilevel framework to reconstruct anatomical 3D models of the hepatic vasculature in rat livers. J Anat. 2017;230(3):471-83.

30. Moschetta M, Mishima Y, Kawano Y, Manier S, Paiva B, Palomera L, et al. Targeting vasculogenesis to prevent progression in multiple myeloma. Leukemia. 2016:30(5):1103-15.

31. Susaki EA, Tainaka K, Perrin D, Kishino F, Tawara T, Watanabe TM, et al. Whole-brain imaging with single-cell resolution using chemical cocktails and computational analysis. Cell. 2014;157(3):726-39.

32. Tainaka K, Kubota SI, Suyama TQ, Susaki EA, Perrin D, Ukai-Tadenuma M, et al. Whole-body imaging with single-cell resolution by tissue decolorization. Cell. 2014;159(4):911-24.

33. Santi PA. Light sheet fluorescence microscopy: a review. J Histochem Cytochem. 2011:59(2):129-38

34. Uzarski J, Drelles MB, Gibbs SE, Ongstad EL, Goral JC, McKeown KK, et al. The resolution of lymphedema by interstitial flow in the mouse tail skin. Am J Physiol Heart Circ Physiol. 2008;294(3):H1326-34.

35. Rousso DL, Qiao M, Kagan RD, Yamagata M, Palmiter RD, Sanes JR. Two pairs of ON and OFF retinal ganglion cells are defined by intersectional patterns of transcription factor expression. Cell Rep. 2016;15(9):1930-44.

36. Susaki EA, Ueda HR. Whole-body and whole-organ clearing and imaging techniques with single-cell resolution: toward organism-level systems biology in mammals. Cell Chem Biol. 2016;23(1):137-57.

37. Kubota SI, Takahashi K, Nishida J, Morishita Y, Ehata S, Tainaka K, et al. Whole-body profiling of Cancer metastasis with single-cell resolution. Cell Rep. 2017:20(1):236-50. 
38. Ueda HR, Ertürk A, Chung K, Gradinaru V, Chédotal A, Tomancak P, et al. Tissue clearing and its applications in neuroscience. Nat Rev Neurosci. 2020;21(2):61-79.

39. Romanov RA, Zeisel A, Bakker J, Girach F, Hellysaz A, Tomer R, et al. Molecular interrogation of hypothalamic organization reveals distinct dopamine neuronal subtypes. Nat Neurosci. 2017;20(2):176-88.

40. Maruoka H, Nakagawa N, Tsuruno S, Sakai S, Yoneda T, Hosoya T. Lattice system of functionally distinct cell types in the neocortex. Science. 2017:358(6363):610-5.

41. Dupraz S, Hilton BJ, Husch A, Santos TE, Coles CH, Stern S, et al. RhoA controls axon extension independent of specification in the developing brain. Curr Biol. 2019;29(22):3874-3886.e3879.

42. Arganda-Carreras I, Fernández-González R, Muñoz-Barrutia A, Ortiz-DeSolorzano C. 3D reconstruction of histological sections: application to mammary gland tissue. Microsc Res Tech. 2010;73(11):1019-29.

43. Matsumaru D, Murashima A, Fukushima J, Senda S, Matsushita S, Nakagata N, et al. Systematic stereoscopic analyses for cloacal development: the origin of anorectal malformations. Sci Rep. 2015;5:13943.

44. Hashimoto D, Kajimoto M, Ueda Y, Hyuga T, Fujimoto K, Inoue S, et al. 3D reconstruction and histopathological analyses on murine corporal body. Reprod Med Biol. 2021;20(2):199-207.

45. Mori Y, Umeda M, Fukunaga M, Ogasawara K, Yoshioka Y. MR contrast in mouse lymph nodes with subcutaneous administration of iron oxide particles: size dependency. Magn Reson Med Sci. 2011;10(4):219-27.

46. Park SY, Bae DS, Nam JH, Park CT, Cho CH, Lee JM, et al. Quality of life and sexual problems in disease-free survivors of cervical cancer compared with the general population. Cancer. 2007;110(12):2716-25.

47. Lockwood-Rayermann S. Lymphedema in gynecologic cancer survivors: an area for exploration? Cancer Nurs. 2007;30(4):E11-8.

48. Omidi Z, Kheirkhah M, Abolghasemi J, Haghighat S. Effect of lymphedema self-management group-based education compared with social network-based education on quality of life and fear of cancer recurrence in women with breast cancer: a randomized controlled clinical trial. Qual Life Res. 2020;29(7):1789-800.

49. Petrek JA, Senie RT, Peters M, Rosen PP. Lymphedema in a cohort of breast carcinoma survivors 20 years after diagnosis. Cancer. 2001;92(6):1368-77.

50. Ogata F, Fujiu K, Matsumoto S, Nakayama Y, Shibata M, Oike Y, et al. Excess Lymphangiogenesis cooperatively induced by macrophages and CD4(+) T cells drives the pathogenesis of lymphedema. J Invest Dermatol. 2016;136(3):706-14.

51. Alitalo K. The lymphatic vasculature in disease. Nat Med. 2011;17(11):1371-80.

52. Saito $Y$, Nakagami $H$, Kaneda Y, Morishita R. Lymphedema and therapeutic lymphangiogenesis. Biomed Res Int. 2013;2013:804675.

53. Jimi S, De Francesco F, Ferraro GA, Riccio M, Hara S. A novel skin splint for accurately mapping dermal remodeling and epithelialization during wound healing. J Cell Physiol. 2017;232(6):1225-32.

54. Iwasaki D, Yamamoto Y, Murao N, Oyama A, Funayama E, Furukawa $H$. Establishment of an acquired lymphedema model in the mouse hindlimb: technical refinement and molecular characteristics. Plast Reconstr Surg. 2017;139(1):67e-78e.
55. Jorgensen MG, Toyserkani NM, Hansen CR, Hvidsten S, Baun C, Hejbol EK, et al. Quantification of chronic lymphedema in a revised mouse model. Ann Plast Surg. 2018:81(5):594-603.

56. Wang Z, Kim KY, Yoon SH, Park JH, Choi J, Bakheet N, et al. Radiation inhibits lymph drainage in an acquired lymphedema mouse Hindlimb model. Lymphat Res Biol. 2020;18(1):16-21.

57. Kwon S, Janssen CF, Velasquez FC, Zhang S, Aldrich MB, Shaitelman SF, et al. Radiation dose-dependent changes in lymphatic remodeling. Int J Radiat Oncol Biol Phys. 2019;105(4):852-60.

58. Zampell JC, Elhadad S, Avraham T, Weitman E, Aschen S, Yan A, et al. Tolllike receptor deficiency worsens inflammation and lymphedema after lymphatic injury. Am J Physiol Cell Physiol. 2012;302(4):C709-19.

59. Tian W, Rockson SG, Jiang X, Kim J, Begaye A, Shuffle EM, et al. Leukotriene $B(4)$ antagonism ameliorates experimental lymphedema. Sci Transl Med. 2017;9(389).

60. Jun H, Lee JY, Kim JH, Noh M, Kwon TW, Cho YP, et al. Modified mouse models of chronic secondary lymphedema: tail and hind limb models. Ann Vasc Surg. 2017;43:288-95.

61. Yao Z, Huang Y, Luo G, Wu J, He W. A biological membrane-based novel excisional wound-splinting model in mice (with video). Burns Trauma. 2014;2(4):196-200.

62. Bai W, Kuang T, Chitrakar C, Yang R, Li S, Zhu D, et al. Patchable micro/nanodevices interacting with skin. Biosens Bioelectron. 2018;122:189-204.

63. Swift T, Westgate G, Van Onselen J, Lee S. Developments in silicone technology for use in stoma care. Br J Nurs. 2020;29(6):S6-s15.

64. White M. Using silicone technology to maintain healthy skin in stoma care. Br J Nurs. 2014;23(22):1188-93.

65. Achouri A, Huchon C, Bats AS, Bensaid C, Nos C, Lécuru F. Complications of lymphadenectomy for gynecologic cancer. Eur J Surg Oncol. 2013;39(1):81-6.

66. Sener SF, Winchester DJ, Martz CH, Feldman JL, Cavanaugh JA, Winchester DP, et al. Lymphedema after sentinel lymphadenectomy for breast carcinoma. Cancer. 2001;92(4):748-52.

67. Keegan KA, Cookson MS. Complications of pelvic lymph node dissection for prostate cancer. Curr Urol Rep. 2011;12(3):203-8.

68. Soga S, Onishi F, Jinzaki M, Mikoshi A, Minabe T, Shinmoto H. Analysis of collateral lymphatic circulation in patients with lower limb lymphedema using magnetic resonance lymphangiography. J Vasc Surg Venous Lymphat Disord. 2021;9(2):471-481.e471.

69. Mitra A, Yan J, Xia X, Zhou S, Chen J, Mishra L, et al. IL6-mediated inflammatory loop reprograms normal to epithelial-mesenchymal transition $(+)$ metastatic cancer stem cells in preneoplastic liver of transforming growth factor beta-deficient $\beta 2$-spectrin(+/-) mice. Hepatology. 2017;65(4):1222-36.

70. Cuzzone DA, Weitman ES, Albano NJ, Ghanta S, Savetsky IL, Gardenier JC, et al. IL-6 regulates adipose deposition and homeostasis in lymphedema. Am J Physiol Heart Circ Physiol. 2014;306(10):H1426-34.

\section{Publisher's Note}

Springer Nature remains neutral with regard to jurisdictional claims in published maps and institutional affiliations.

\footnotetext{
Ready to submit your research? Choose BMC and benefit from:

- fast, convenient online submission

- thorough peer review by experienced researchers in your field

- rapid publication on acceptance

- support for research data, including large and complex data types

- gold Open Access which fosters wider collaboration and increased citations

- maximum visibility for your research: over 100M website views per year
}

At BMC, research is always in progress.

Learn more biomedcentral.com/submissions 